\section{A Comparison Between Direct and Indirect Friction Measurements in a Forced Oscil- lator}

\author{
J.-W. Liang \\ Department of Mechanical Engineering \\ MingChi Institute of Technology \\ Taipei, Taiwan, R.O.C.

\section{B. F. Feeny \\ Department of Mechanical Engineering Michigan State University East Lansing, MI 48824}

\section{Journal of Applied Mechanics 65 (3) 783-786.}

\section{Introduction}

Characterizing macroscopic low-order friction behavior is important in many engineering tasks, such as those involving control, and squeak and squeal prediction. Two schools of thought for measuring macroscopic friction forces involve the employment of a load cell and the calculation of friction force by measuring motion signals and applying them to the system's governing equation. Direct load-cell measurements are most often found in the literature. Examples can be found in recent books and reviews, such as Guran et al. (1996), Ibrahim (1994), Armstrong-Hélouvry et al. (1994), and Oden and Martins (1985), or in any journal on the topic.

Installing a load cell to frictional systems is equivalent to adding a mass-spring subsystem and its dynamics to the main system (Streator and Bogy, 1994). Thus, the signal obtained from a load cell may not be able to completely depict actual friction forces. On the other hand, the "indirect" calculation of the friction force from the system's ordinary differential equation (ODE) requires more than one transducer. This approach is therefore liable to parasitic interference and calibration errors (Antoniou et al., 1965). Moreover, a smoothening algorithm is often required to handle digital data (e.g. Sakamoto, 1987; and Dupont and Dunlap, 1995) and may affect the apparent friction characteristics.

A brief comparison between friction signals from both direct and indirect friction measurements is presented in this letter. The measurements displayed here are in a forced mass-spring system during macroscopic sliding and macroscopic stick-slip. More details on the classification and modeling of these motions for this system were given previously (Liang and Feeny, 1996).

\section{Apparatus and Instrumentation}

The apparatus (Figure 1) consisted of a base-excited mass ( $m=$ $2.42 \mathrm{~kg}$ ), helical springs with a total stiffness of $k=2310 \mathrm{~N} / \mathrm{m}$, and the friction-contact mechanism. The sliding mass moved in an air track. Motion in the air track was almost friction free, with a nondi- mensional viscous damping factor equal to 0.0008 . This damping is subsequently neglected. The friction-contact mechanism consisted of a pinched-flange structure which was designed for balancing the normal loads on both sliding surfaces.

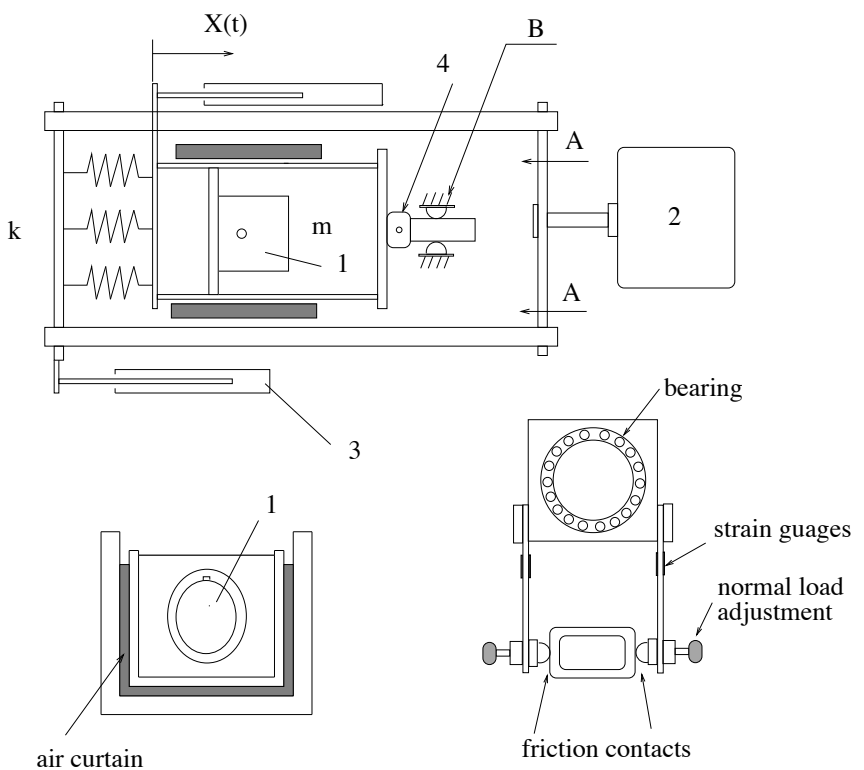

A-A sectional view

details in portion $\mathrm{B}$

1 : accelerometer

2 : shaker

3 : LVDT

4 : force transducer

Figure 1: Schematic diagram of the experimental apparatus.

The displacements of both the sliding mass and the base excitation were sensed by linear variable differential transformer (LVDT). The LVDT signal passed through a signal conditioner. This LVDT was made by Rabinson-Halpern Co. (Model 210A-0500) and had a resolution of $2.5 \mu \mathrm{m}$ after quantization. A seismic accelerometer (PCB, Model 393C) was adopted to record the acceleration signal. This accelerometer had a frequency range of $0.025 \mathrm{~Hz}$ to $800 \mathrm{~Hz}$ with $5 \%$ transverse sensitivity and a resonant frequency of $3.5 \mathrm{kHz}$ (125 Hz with its mounting). The friction force was measured by a piezoelectric load cell (PCB, Model 208B) which had $0.0002 \mathrm{lb}$ resolution in a range of $10 \mathrm{lb}$ in both tension and compression. The nominal sensitivity was $500 \mathrm{mV} / \mathrm{lb}$ with a stiffness of $10 \mathrm{lb} / \mu \mathrm{in}$. The discharge time constant of this load cell was $50 \mathrm{sec}$ and the resonant frequency was $70 \mathrm{kHz}(250 \mathrm{~Hz}$ with its attachment). The sampling rate was $5 \mathrm{kHz}$.

The system was driven by an electromagnetic shaker (LDS, Model 400). The friction contact was steel-on-steel. The planar surfaces were ground and rubbed with 400-grit, silicon-carbide paper. The other contact surfaces had hemispherical geometry and were lathed with an engineering finish then rubbed by the same type of paper. Finally, the surfaces were cleaned by a degreaser (Measurement Group, Inc., Model CSM-1). The surfaces were then engaged in sliding motion for at least 30 minutes to attain a steady-state fric- 
tion characteristic before the data were recorded.

To investigate the phase shift between signals from the accelerometer and LVDT, a free-vibration test of the mass-spring system was conducted with the base constrained.

The measurements of displacement and acceleration are labeled as $S_{1}(t)$ and $S_{2}(t)$ respectively. There was a phase shift of $\phi=0.0389$ radians by which the accelerometer signal leads the LVDT signal. This phase angle was converted to a time shift using $\tau=\phi / \omega_{n}$, and it was chosen such that the resultant force, namely $m S_{2}(t+\tau)+k S_{1}(t)$, was close to zero with some random noise. The random noise could be induced by the sound and the pressure fluctuation of the air track or another source.

Three sets of base-excited motion tests (Liang, 1996) indicated that the dependence of the phase shift on the excitation frequency was not significant over a reasonable range. The phase angles of accelerometer and the load cell were presumed to be approximately equal to zero based on their response characteristics and the lowfrequency range of this study.

\section{The Comparison between Friction Signals}

To calculate the friction force from the system's equation of motion, namely $F(t)=k S_{3}(t)-k S_{1}(t)-m S_{2}(t+\tau)$, where $S_{3}(t)$ represents the motion of the base, motion signals $S_{1}(t), S_{2}(t)$, and $S_{3}(t)$ are required.

Figure 2(a) illustrates the time-domain histories of the experimental inertial forces, $m S_{2}(t+\tau)$, spring force, $k S_{1}(t)$, and the base-excitation force, $k S_{3}(t)$. The frequency of harmonic excitation is $5.5 \mathrm{~Hz}$. The response of the slider is a stable pure-sliding motion. The calculated friction force is presented in Figure 2(b) and denoted as $\mathrm{F}(\mathrm{t})$. Random noise caused mostly by the air track was superimposed on the calculated friction signal. To smoothen the signal, a five-point moving average (Liang, 1996) was applied to generate the data shown in Figure 2(c) and labeled F1(t). Next, the friction force obtained from the direct measurements of the load cell after the same smoothening process (for comparison) is illustrated in Figure 2(d) as F2(t). The signal F2(t) is obtained by subtracting the inertial component on the load cell due to the mass of the flange from the readout of the load cell. The raw version of F2(t) is very similar to the averaged version since it does not register much transient dynamics as in the computation case. An FFT of these two signals prior to smoothening is shown in Figure 3.

Three observations are made from these Figures. (1) During the whole test, the friction-force magnitudes are more-or-less constant. (2) Both methods are consistent regarding the macroscopic dynamics friction feature. (3) There are subtle differences between the two approaches at the change in sliding direction. Regarding observation (3), a higher-frequency dynamical response is registered in the calculated friction force than in the directly measured friction force. This is probably because the load cell, with its attached flange, acts as a low-pass filter, and attenuates high-frequencies. Furthermore, some high-frequency oscillation is evident in the calculated friction signal immediately following a velocity reversal. We speculate that this may be due to the dynamics of the accelerometer mounting.

Two other stable pure-sliding cases were examined in which the excitation frequencies were 3.5 and $7.5 \mathrm{~Hz}$ (Liang, 1996). Consis- (a)

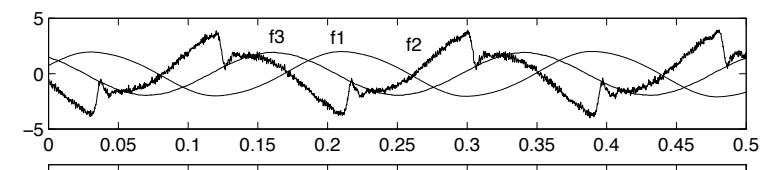

(b)

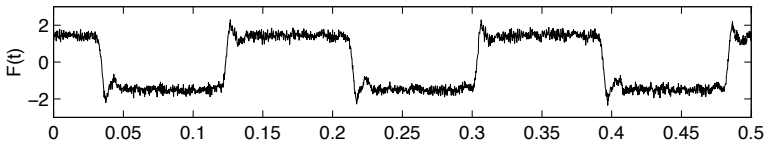

(c)

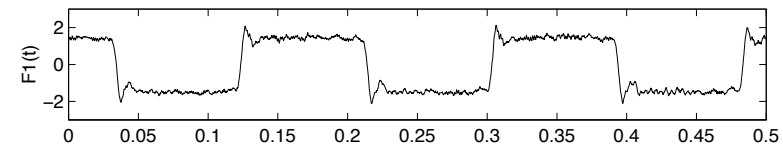

(d)

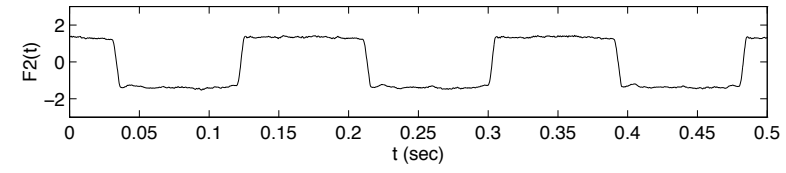

Figure 2: Time domain comparison of direct and indirect friction measurements, excitation frequency $=5.5 \mathrm{~Hz}$, stable pure sliding case, in (a) f1 labels the experimental spring force $=k S_{1}(t)$; $\mathrm{f} 2$ labels the experimental inertial force $=$ $m S_{2}(t+\tau)$; f3 labels the experimental excitation force $=$ $k S_{3}(t)$; (b) $\mathrm{F}(\mathrm{t})$ is the calculated friction force; (c) $\mathrm{F} 1(\mathrm{t})$ is the averaged version of $F(t)$; (d) F2(t) represents the load cell measurement after averaging.

tencies and discrepancies between the two approaches are preserved in these tests, with the post reversal oscillation slightly more prominent in the indirect signal of the latter case, and less evident in the former case. Since the same phase relationship was employed between the sensors at each excitation frequency, the consistent results indeed illustrate the reliability of the indirect approach over a reasonable frequency range.

Figure 4 illustrates a case that has stick-slip motion. The excitation frequency is $3.5 \mathrm{~Hz}$. Due to the presence of stick-slip, features of the friction force are different, especially during the transition of sliding to sticking. A $47-\mathrm{Hz}$ transition oscillation occurs during the sticking phase. This transition oscillation is likely caused by the stiffness of the contact and its surrounding structure (Liang and Feeny, 1996).

\section{Conclusion}

In this study, we compared friction measurements computed from motion sensors with those obtained directly from a load cell. Comparisons of these techniques showed that, although load cell registered most of the friction dynamics, its high-frequency contents were attenuated to some extent. The calculated friction may have caught more complete details over the low and high frequency ranges and also captured the noise generated by the air track and possibly the dynamics of the mounting.

While there are high-frequency differences between the direct and indirect measurements, the the correlation in the results provides a cross reference and suggests that the load cell can be trusted for the low-order friction behavior. For simple oscillators, 

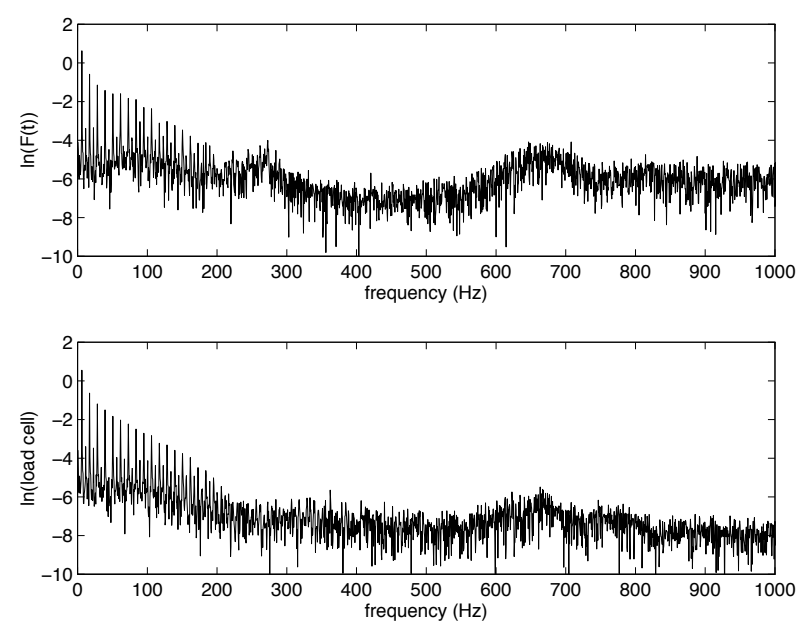

Figure 3: Frequency domain comparison of direct and indirect friction measurements, excitation frequency $=5.5 \mathrm{~Hz}$, stable pure sliding case. The upper and lower plots represent the indirect and load-cell measurements prior to smoothening.

which may be useful in studying basic phenomena such as friction, it seems that either measurement method would be suitable. In more general multi-degree-of-freedom systems, the indirect measurement based on measured states is impractical, as the number of measurements needed may be arbitrarily large, and knowledge of the system equations of motion may be limited.

\section{ACKNOWLEDGEMENTS}

Years ago, Andy Ruina suggested estimating the friction force indirectly from the equations of motion. This work was supported in part by the National Science Foundation and Ford Motor Company.

\section{REFERENCES}

Antoniou, S. S., Cameron, A., and Gentle, C. R., 1976, "The friction-speed relationship from stick-slip data," Wear 36 235-254.

Armstrong-Hélouvry, B., Dupont, P., and Canudas de Wit, C., 1994, "A survey of models, analysis tools and compensation methods for the control of machines with friction," Automatica 30 (7) 1083-1138.

Sakamoto, T., 1987, "Normal displacement and dynamical friction characteristics in a stick-slip process," Tribology International 20 (1) 25-31.

Dupont, P. E and Dunlap, E. P., 1995, "Friction modeling and PD compensation at very low velocities," Journal of Dynamic Systems, Measurement, and Control 117 8-14.

Guran, A., Pfeiffer, F., and Popp, K., 1996, Dynamics with Friction: Modeling, Analysis and Experiment. Part I, World Scientific, River Edge.

Ibrahim, R. A., 1994, "Friction-induced vibration, chatter, squeal, and chaos: Part I-mechanics of friction; Part II - dynamics (a)

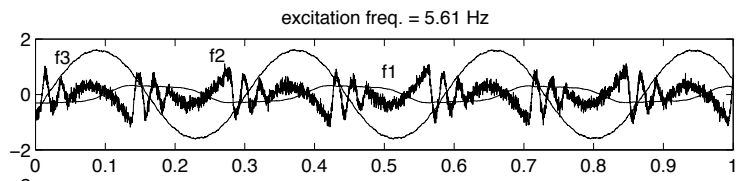

(b)

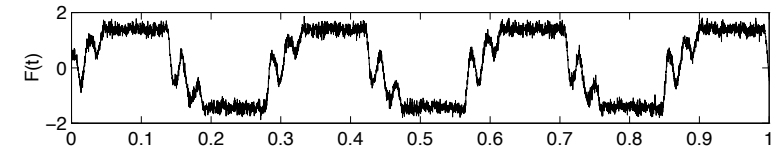

(c)

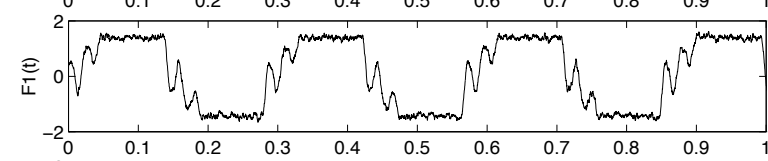

(d)

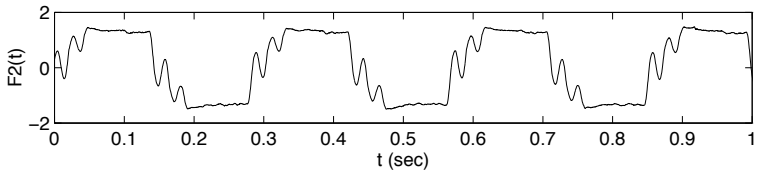

Figure 4: Time domain comparison of direct and indirect friction measurements, excitation frequency $=3.5 \mathrm{~Hz}$, stickslip motion case, in (a) $\mathrm{f} 1$ : the experimental spring force $=$ $k S_{1}(t)$; 2 : the experimental inertial force $=m S_{2}(t+\tau)$; $\mathrm{f} 3$ : the experimental excitation force $=k S_{3}(t)$; (b) $\mathrm{F}(\mathrm{t})$ is the calculated friction force; $(\mathrm{c}) \mathrm{F} 1(\mathrm{t})$ is the averaged version of $\mathrm{F}(\mathrm{t})$; (d) F2(t) represents the load cell measurement after averaging.

and modeling," Applied Mechanics Reviews 47 (7) 209-253.

Liang, J.-W., 1996, Characterizing the Low-Order Friction Dynamics in a Forced Oscillator, PhD thesis, Michigan State University, East Lansing.

Liang, J.-W. and Feeny, B. F., 1996, "The effects of tangential contact stiffness on a harmonically forced oscillator," Proceedings of the symposium on Elasto-Impact and Friction in Dynamic Systems, ASME International Congress and Exhibition, Atlanta.

Oden, J. T., and Martins, J. A. C., 1985, "Models and computational methods for dynamic friction phenomena," Computer Methods in Applied Mechanics and Engineering 52 527-634.

Streator, J. L. and Bogy, D. B., 1992, "Accounting for transducer dynamics in the measurement of friction," Journal of Tribology 114 86-94. 